九州大学学術情報リポジトリ

Kyushu University Institutional Repository

\title{
IMPLICATION AND FUNCTIONAL DEPENDENCY IN INTENSIONAL CONTEXTS
}

Ishida, Toshikazu

Department of Informatics, Kyushu University

Honda, Kazumasa

Department of Informatics, Kyushu University

Kawahara, Yasuo

Department of Informatics, Kyushu University

https://doi.org/10.5109/18997

出版情報: Bulletin of informatics and cybernetics. 40, pp.101-111，2008-12. Research Association of Statistical Sciences

バージョン :

権利関係 : 


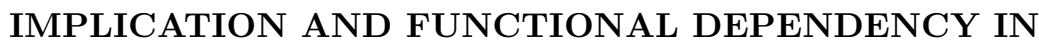

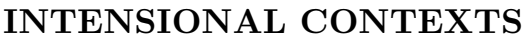

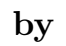

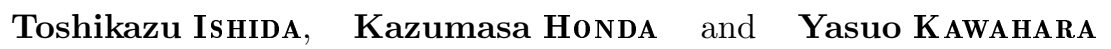

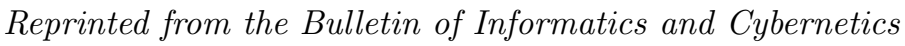

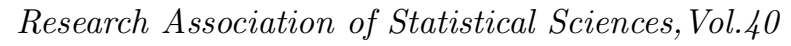

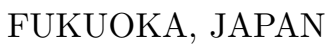

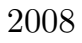




\title{
IMPLICATION AND FUNCTIONAL DEPENDENCY IN INTENSIONAL CONTEXTS
}

\author{
By
}

\author{
Toshikazu IshidA, Kazumasa HoNDA ${ }^{\dagger}$ and Yasuo KawaHARA ${ }^{\ddagger}$
}

\begin{abstract}
Formal concept analysis is a mathematical field applied to data mining. Usually, a formal concept is defined as a pair of sets, called extents and intents, for a given formal context in binary relation. In this paper we review the idea that Armstrong's inference rules are complete and sound for functional dependencies. Then, we prove that Armstrong's inference rules are complete and sound for implications of formal contexts. Still, we give an example which shows the difference between implication and functional dependency. Besides, we show that functional dependency can be reduced to implication. Finally, we give the condition on which a set of implications and a set of functional dependencies for the intensional context are equivalent.
\end{abstract}

Key Words and Phrases: Formal concept, Functional dependency, Implication, Armstrong inference rules

\section{Introduction}

In the philosophical theory, a concept is defined as a pair of an extent and an intent. The extent is a subset of all objects that belong to the concept, and the intent is a subset of all attributes whose object are common. In the mathematical theory, formal concepts mean formal models of concepts as defined above. Our subjects are the correlations between formal concepts. The method to analyze them is called formal concept analysis. Based on the lattice theory, it was proposed by Wille in 1970's. The standard textbook is Ganter and Wille (1999).

What we call a formal context is really a database which consists of a set of objects, a set of attributes and the binary relation between them. The formal context defines formal concepts as suitable pairs of an extent and an intent. The idea is an extension of Dedekind cuts of real numbers. The set of all formal concepts forms a complete lattice by inclusion between extents. The complete lattice implies the features of a formal context. Therefore, formal concept analysis is applicable to data mining.

To find a correlation of attributes is important for analyzing a relational database. Codd (1970) introduced a notion of functional dependency, which is a constraint between two sets of attributes. Armstrong (1974) proposed so-called Armstrong inference rules. Beeri, Fagin and Howard (1977) proposed that those inference rules are complete and sound for functional dependencies. On the other hand, Ganter and Wille defined implication, or another type of dependency in a formal context.

\footnotetext{
* Department of Informatics, Kyushu University, Fukuoka, 819-0395, Japan. t-ishida@i.kyushu-u.ac.jp

$\dagger$ Department of Informatics, Kyushu University

$\ddagger$ Department of Informatics, Kyushu University
} 
In this paper, we review a simple proof that Armstrong inference rules are sound and complete for functional dependencies in formal contexts. Also we demonstrate that Armstrong inference rules are sound and complete for implications for formal contexts. Further, to distinguish semantics and syntax, we give a common proof of implication and functional dependency. Still, we give an example which shows the difference between implication and functional dependency.

The paper is organized as follows. In Section 2 we introduce an intensional context which could be discussed more clearly than a formal context. In Section 3 we review Armstrong inference rules for the dependency of attributes. In section 4 we examine the functional dependency in intensional context, and show that Armstrong inference rules are sound for functional dependencies. In Section 5 we explain a notion of implication in intensional contexts, and then prove some properties of implication to show that Armstrong inference rules are sound for it. In Section 6 we demonstrate that Armstrong inference rules are complete for implications and functional dependencies. Still, in Section 7, we notice an example which shows the difference between implication and functional dependency.

\section{Intensional Contexts}

A formal context is a binary relation between objects and attributes. The following shows a simple example of formal contexts.

\begin{tabular}{|c|c|c|c|}
\hline & $a$ & $b$ & $c$ \\
\hline$x_{0}$ & $\times$ & $\times$ & \\
\hline$x_{1}$ & & $\times$ & $\times$ \\
\hline$x_{2}$ & & & $\times$ \\
\hline$x_{3}$ & & $\times$ & $\times$ \\
\hline
\end{tabular}

Where $a, b$ and $c$ are attributes, $x_{0}, x_{1}, x_{2}$ and $x_{3}$ objects. The formal concept lattice $C$ is:

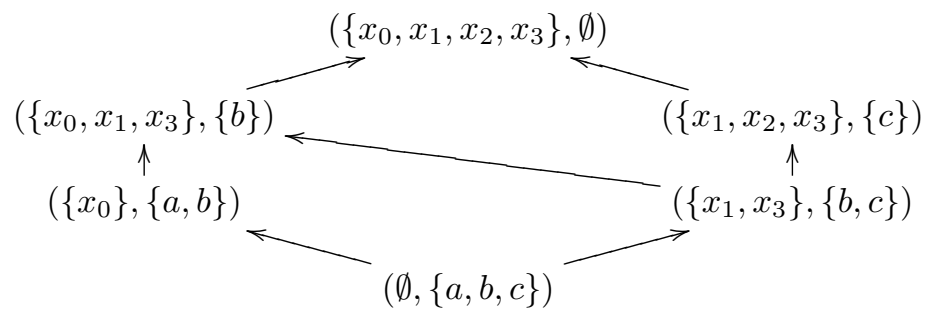

Construct the family $\mathcal{T}=\{\{a, b\},\{b, c\},\{c\}\}$ of the subsets of attributes related with each object, and the closure system

$$
\mathcal{T}^{*}=\{\{a, b, c\},\{a, b\},\{b, c\},\{b\},\{c\}, \emptyset\}
$$

generated by $\mathcal{T}$. It is easy to see that $\mathcal{T}^{*}$ considers with the set of all intents (second components) of $C$. For constructing concept lattices, it is enough to treat with a family of subsets of attributes instead of a formal context. In this paper, we argue by using such framework.

Definition 2.1. Let $Y$ be a set of attributes and $\wp(Y)$ the power set of $Y$. A subset $\mathcal{T}$ of $\wp(Y)$ is called an intensional context on $Y$. 


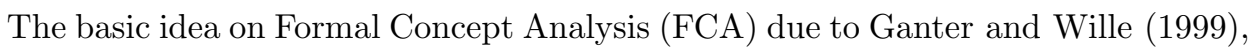

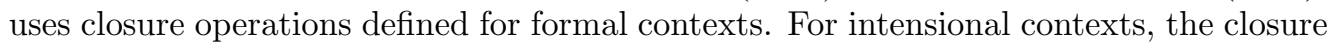

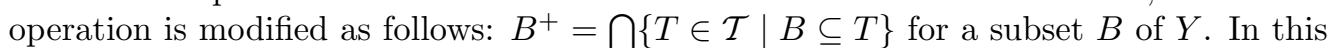

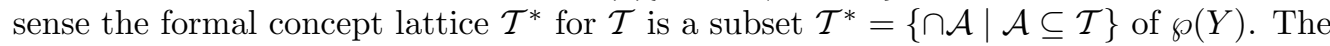

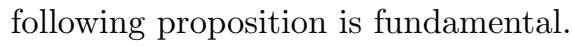

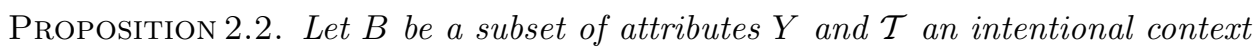

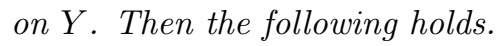

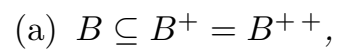

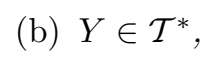

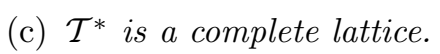

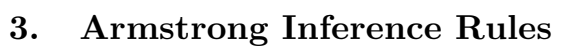

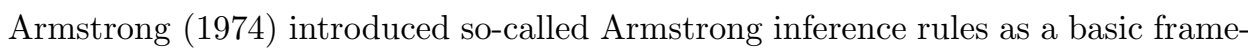

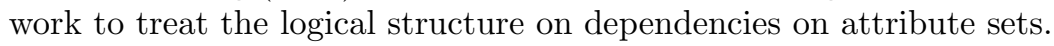

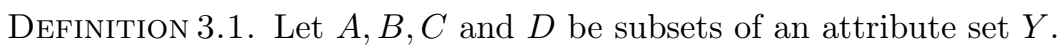

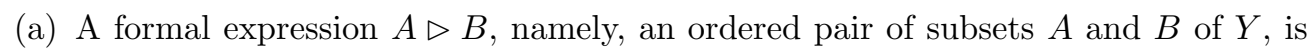

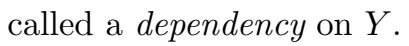

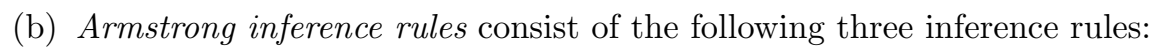

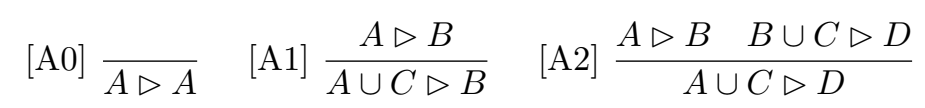

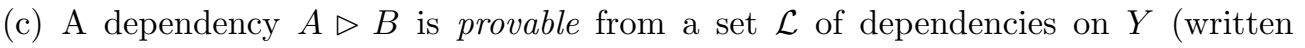

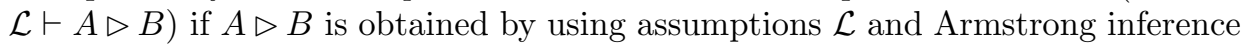

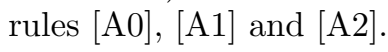

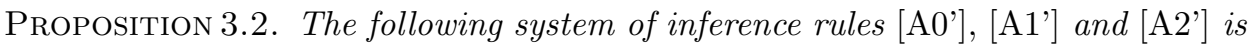

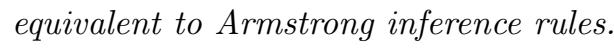

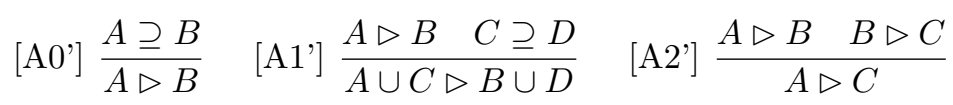

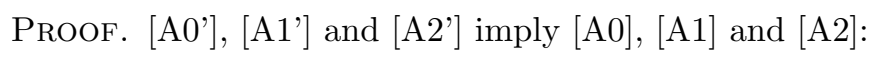

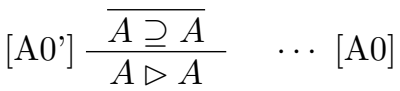

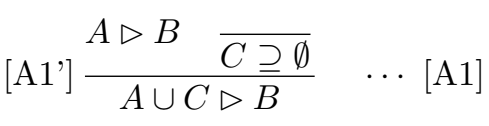

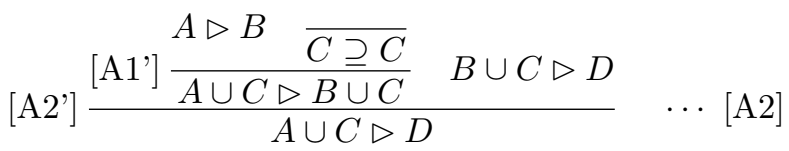




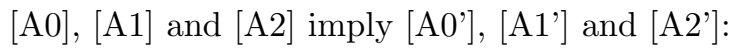

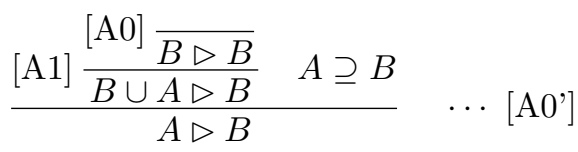

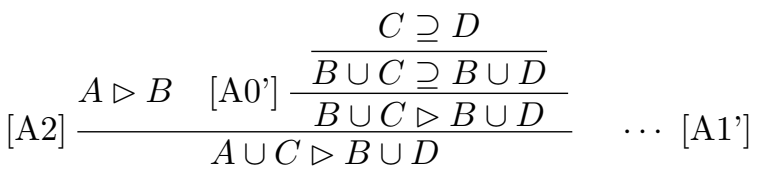

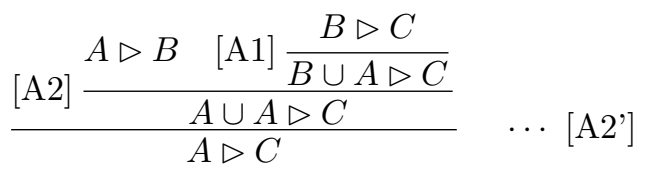

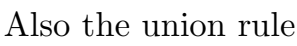

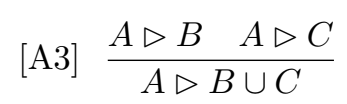

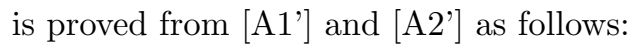

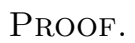

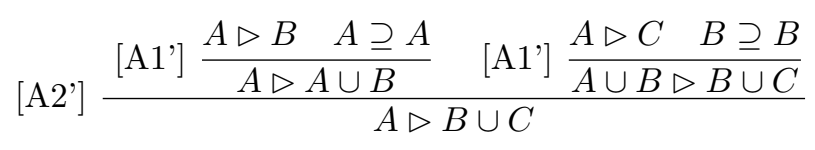

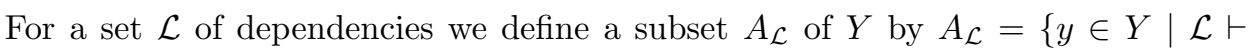

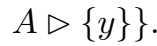

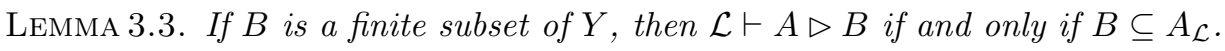

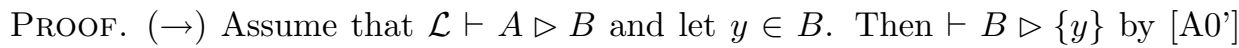

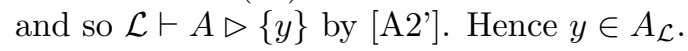

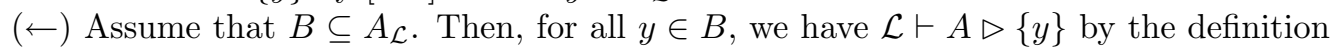

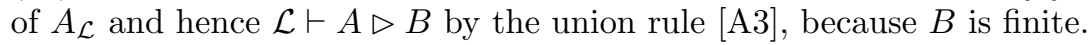

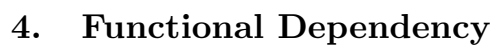

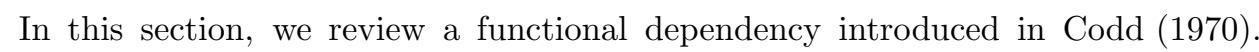

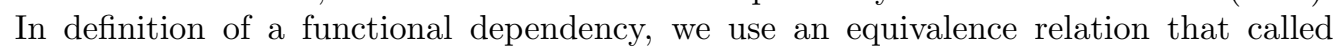

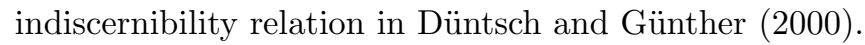

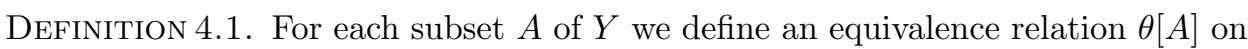

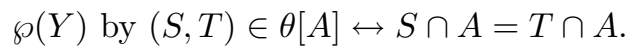

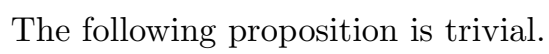

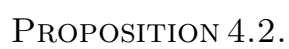

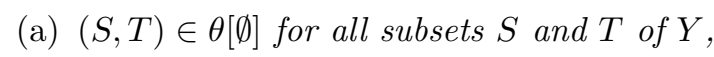



$\left(\mathrm{A} 0^{\prime}\right),\left(\mathrm{A} 1^{\prime}\right)$ and $\left(\mathrm{A} 2^{\prime}\right)$ are called reflexive law, augmentation law and transitive law, respectively.

The following proposition will be used in our proof of the completeness of Armstrong inference rules for functional dependencies.

Proposition 4.6. Let $A$ be a proper subset of $Y$. There exists a set $\mathcal{T}_{0}$ such that

(a) $C \subseteq A$ if and only if $\mathcal{T}_{0} \models_{F} \emptyset \triangleright C$,

(b) $C \nsubseteq A$ if and only if $\mathcal{T}_{0}=_{F} C \triangleright Y$.

ProOF. (a)

$$
\begin{aligned}
\mathcal{T}_{0}={ }_{F} \emptyset \triangleright C & \leftrightarrow(A, Y) \in \theta[\emptyset] \rightarrow(A, Y) \in \theta[C] & & \{4.4\} \\
& \leftrightarrow(A, Y) \in \theta[C] & & \{4.2(\mathrm{a})\} \\
& \leftrightarrow A \cap C=Y \cap C=C & & \{C \subseteq Y\} \\
& \leftrightarrow C \subseteq A . & &
\end{aligned}
$$

(b)

$$
\begin{array}{rlll}
\mathcal{T}_{0} \mid={ }_{F} C \triangleright Y & \leftrightarrow(A, Y) \in \theta[C] \rightarrow(A, Y) \in \theta[Y] & & \{4.4\} \\
& \leftrightarrow(A, Y) \notin \theta[C] \vee A=Y & & \{4.2(\mathrm{~b})\} \\
& \leftrightarrow(A, Y) \notin \theta[C] & & \{A \neq Y\} \\
& \leftrightarrow C \nsubseteq A . & & \{(\mathrm{a})\}
\end{array}
$$

Proposition 4.7. Let $\mathcal{T}$ be an intensional context on $Y$ and $a, b \in Y$. If $\mathcal{T} \models_{F}$ $\{a\} \triangleright\{b\}$ and $\mathcal{T} \not \models_{F}\{b\} \triangleright\{a\}$, then $\mathcal{T} \models_{F} \emptyset \triangleright\{b\}$.

Proof. Since $\mathcal{T} \forall_{F}\{b\} \triangleright\{a\}$, there exist $S_{0}, T_{0} \in \mathcal{T}$ such that $a \in S_{0}, a \notin T_{0}$ and $\{b\} \cap S_{0}=\{b\} \cap T_{0}$. Consider $\mathcal{T}_{a}=\{S \in \mathcal{T} \mid a \in S\}$ and $\mathcal{T}_{\neg a}=\{S \in \mathcal{T} \mid a \notin S\}$. Then $\{b\} \cap S=\{b\} \cap S_{0}$ and $\{b\} \cap T=\{b\} \cap T_{0}$ for all $S \in \mathcal{T}_{a}$ and all $T \in \mathcal{T}_{\neg a}$, respectively, because $\mathcal{T} \models_{F}\{a\} \triangleright\{b\}$. Hence, for all $S \in \mathcal{T}_{a}$ and $T \in \mathcal{T}_{\neg a},\{b\} \cap S=\{b\} \cap S_{0}=$ $\{b\} \cap T_{0}=\{b\} \cap T$. And then $\{b\} \cap S=\{b\} \cap T$ for all $S, T \in \mathcal{T}$. Therefore we have $\mathcal{T} \models{ }_{F} \emptyset \triangleright\{b\}$.

\section{Implication}

In this section, we review implication introduced in Ganter and Wille (1999).

Definition 5.1. Let $\mathcal{T}$ be an intensional context on $Y, A \triangleright B$ a dependency on $Y$ and $\mathcal{L}$ a set of dependencies on $Y$. We define two notations $\mathcal{T} \models_{I} A \triangleright B$ and $\mathcal{T} \models_{I} \mathcal{L}$ as follows:

(a) $\mathcal{T} \models_{I} A \triangleright B$ if $A \subseteq T$ implies $B \subseteq T$ for all $T \in \mathcal{T}$,

(b) $\mathcal{T} \models_{I} \mathcal{L}$ if $\mathcal{T} \models_{I} A \triangleright B$ for all $A \triangleright B \in \mathcal{L}$.

If $\mathcal{T}={ }_{I} A \triangleright B$ then $A \triangleright B$ is valid (as implication) for an intensional context $\mathcal{T}$ on $Y$ or is called an implication on $\mathcal{T}$. A set of implication $\mathcal{L}$ is valid for $\mathcal{T}$ on $Y$ if $\mathcal{T} \models_{I} \mathcal{L}$.

The next proposition means that Armstrong inference rules are sound for implications. 
Proposition 5.2. Let $\mathcal{T}$ be an intensional context on $Y, A \triangleright B$ a dependency on $Y$, and $L$ a set of dependencies.

$(\mathrm{A} 0) \mathcal{T} \models{ }_{I} A \triangleright A$,

(A1) If $\mathcal{T} \models_{I} A \triangleright B$ then $\mathcal{T}=_{I} A \cup C \triangleright B$,

(A2) If $\mathcal{T} \models_{I} A \triangleright B$ and $\mathcal{T} \models_{I} B \cup C \triangleright D$ then $\mathcal{T} \models_{I} A \cup C \triangleright D$.

Proof. (A0) It is trivial.

(A1) For all $T \in \mathcal{T}$ we have

$$
\begin{aligned}
A \cup C \subseteq T & \rightarrow A \subseteq T \\
& \rightarrow B \subseteq T . \quad\left\{\mathcal{T} \models_{I} A \triangleright B\right\}
\end{aligned}
$$

(A2) For all $T \in \mathcal{T}$ we have

$$
\begin{aligned}
(A \subseteq T \rightarrow B \subseteq T) & \rightarrow(A \cup C \subseteq T \rightarrow B \cup C \subseteq T) \\
& \rightarrow(A \cup C \subseteq T \rightarrow D \subseteq T) . \quad\left\{\mathcal{T} \models_{I}(B \subseteq C \triangleright D)\right\}
\end{aligned}
$$

The following proposition will be used in our proof of the completeness of Armstrong inference rules for implications.

Proposition 5.3. Let $A$ be a proper subset of $Y$. There exists a set $\mathcal{T}_{0}$ such that

(a) $C \subseteq A$ if and only if $\mathcal{T}_{0} \models{ }_{I} \emptyset \triangleright C$,

(b) $C \nsubseteq A$ if and only if $\mathcal{T}_{0} \models_{I} C \triangleright Y$.

Proof. (a) $\mathcal{T}_{0}=_{I} \emptyset \triangleright C \leftrightarrow(\emptyset \subseteq A \rightarrow C \subseteq A) \leftrightarrow C \subseteq A$.

(b) $T \models_{I} C \triangleright Y \leftrightarrow(C \subseteq A \rightarrow Y \subseteq A) \leftrightarrow C \nsubseteq A$.

Proposition 5.4. Let $\mathcal{T}$ be an intensional context and $A \triangleright B$ a dependency. Then

(a) $\mathcal{T} \models_{I} A \triangleright B$ if and only if $\mathcal{T}^{*} \models_{I} A \triangleright B$,

(b) $\mathcal{T} \models_{I} A \triangleright B$ if and only if $B \subseteq A^{+}$.

ProOF. (a)

$$
\begin{aligned}
\left.\mathcal{T}\right|_{I} A \triangleright B & \leftrightarrow \forall T \in \mathcal{T} \cdot(A \subseteq T \rightarrow B \subseteq T) \\
& \leftrightarrow \forall \mathcal{S} \subseteq \mathcal{T} \cdot \forall T \in \mathcal{S} .(A \subseteq T \rightarrow B \subseteq T) \\
& \leftrightarrow \forall \mathcal{S} \subseteq \mathcal{T} \cdot(A \subseteq \cap \mathcal{S} \rightarrow B \subseteq \cap \mathcal{S}) \\
& \leftrightarrow \mathcal{T}^{*} \models_{I} A \triangleright B .
\end{aligned}
$$

(b)

$$
\begin{aligned}
\mathcal{T} \mid{ }_{I} A \triangleright B & \leftrightarrow \forall T \in \mathcal{T} .(A \subseteq T \rightarrow B \subseteq T) \\
& \leftrightarrow B \subseteq \cap\{T \in \mathcal{T} \mid A \subseteq T\} \\
& \leftrightarrow B \subseteq A^{+} .
\end{aligned}
$$



Then, the following theorem holds.

Theorem 6.3 MaIer. A set $\mathcal{L}$ of dependencies is closed if and only if $\mathcal{L}$ satisfies the following.

(a) $A \triangleright A \in \mathcal{L}$,

(b) if $A \triangleright B \in \mathcal{L}$ then $A \cup C \triangleright B \in \mathcal{L}$,

(c) if $A \triangleright B, B \cup C \triangleright D \in \mathcal{L}$ then $A \cup C \triangleright D \in \mathcal{L}$.

Proof. $(\leftarrow)$ is trivial by structural induction. $(\rightarrow)$

(a)

$$
\begin{array}{rlrl}
\text { true } & \rightarrow \mathcal{L} \vdash A \triangleright A & & \{[\mathrm{~A} 0]\} \\
& \rightarrow A \triangleright A \in \mathcal{L} . & \{\mathcal{L}: \text { closed }\}
\end{array}
$$

(b)

$$
\begin{array}{rlrl}
A \triangleright B \in \mathcal{L} & \rightarrow \mathcal{L} \vdash A \triangleright B & \\
& \rightarrow \mathcal{L} \vdash A \cup C \triangleright B & & \{[\mathrm{~A} 1]\} \\
& \rightarrow A \cup C \triangleright B \in \mathcal{L} . \quad & \{\mathcal{L}: \text { closed }\}
\end{array}
$$

(c)

$$
\begin{aligned}
A \triangleright B, B \cup C \triangleright D \in \mathcal{L} & \rightarrow \mathcal{L} \vdash A \triangleright B \wedge \mathcal{L} \vdash B \cup C \triangleright D & & \\
& \rightarrow \mathcal{L} \vdash A \cup C \triangleright D & & \{[\mathrm{~A} 2]\} \\
& \rightarrow A \cup C \triangleright D \in \mathcal{L} . & & \{\mathcal{L}: \text { closed }\}
\end{aligned}
$$

Conversely assume that $\mathcal{L}$ satisfies the conditions (a), (b) and (c). Recall the completeness theorem

$$
\mathcal{L} \vdash A \triangleright B \text { if and only if } \forall T .\left(T \models_{I} \mathcal{L} \rightarrow T \models_{I} A \triangleright B\right) .
$$

If $\forall T$. $\left(T \models_{I} \mathcal{L} \rightarrow T \models_{I} A \triangleright B\right)$, then $\mathcal{L} \vdash A \triangleright B$ by the completeness theorem and so $A \triangleright B \in \mathcal{L}$ by the conditions (a), (b) and (c).

Therefore, Armstrong Inference rules are sound and complete for functional dependencies and implications.

\section{Difference between Implication and Functional Dependency}

In this section, we show the difference between an implication and a functional dependency, by using examples.

Let an intensional context $\mathcal{T}$ be $\{\{a, b\},\{b, c\},\{c\}\}$.

\begin{tabular}{|c|c|c|c|}
\hline & $a$ & $b$ & $c$ \\
\hline$x$ & $\times$ & $\times$ & \\
\hline$y$ & & $\times$ & $\times$ \\
\hline$z$ & & & $\times$ \\
\hline
\end{tabular}

We consider about the dependency $\{a\} \triangleright\{b\}$. The set $\{a\}^{+}$is $\{a, b\}$. Therefore $\mathcal{T} \models_{I}\{a\} \triangleright\{b\}$. But, for $\{b, c\},\{c\} \in \mathcal{T},\{b, c\} \cap\{a\}=\{c\} \cap\{a\}$, and $\{b, c\} \cap\{b\} \neq$ 



\section{Summary and Outlook}

In this paper, we review the idea that Armstrong inference rules are sound and complete for functional dependencies. Then, we prove that Armstrong inference rules are sound and complete for implications of formal contexts. Further, to distinguish the semantic and syntax, we give a proof which is different from that of Ganter and Wille (1999). In the proof, we ordered the thing of semantic or syntax theory. Still, we give an example which shows the difference between implication and functional dependency. Besides, we show that functional dependency can be reduced to implication. Finally, we give the condition that a set of implications and a set of functional dependencies for a intensional context are equivalent.

In the future, we will consider other conditions on which implication and functional dependency are equivalent.

\section{References}

Ganter, B., Wille, R. (1999). Formal Concept Analysis, Springer-Verlag.

Codd, E. (1970). A relational model of data for large shared data banks, Communications of the ACM 13. 377-387

Armstrong, W. W. (1974). Dependency structures in data base relationships, IFIP Congress, Geneva, Switzerland. 580-583

Beeri, C., Fagin, R., Howard, J.H. (1977). A complete axiomatization for functional and multivalued dependencies in database relations, Proceedings of the $1977 \mathrm{ACM}$ SIGMOD International Conference on Management of Data, Toronto, Canada. $47_{-}$ 61

Düntsch, I., Günther, G. (2000). Rough set data analysis. A road to non-invasive knowledge discovery, Methoঠos Publishers

Received June 24, 2008

Revised October 6, 2008 